\title{
Über hochbasische Lithiumsalze schwacher anorganischer Säuren.
}

\section{Von Artedr Rosenheim und Werner Reguin. ${ }^{1}$ )}

Das zwiespältige Verhalten der Lithiumsalze, die in ihren Eigenschaften teilweise den Alkalisalzen, teilweise aber den Erdalkalisalzen sich anschlieben, ist wohlbekannt und vielfach untersucht. Während die Lithiumsalze meist starker Säuren, wie z. B. der Chlor-, Brom-, Jodwasserstoffsäure, der Salpetersäure und Schwefelsäure den anderen Alkalisalzen entsprechend leichter löslich sind als diese und einen positiven Temperaturkoeffizienten der Löslichkeit besitzen, sind das Lithiumfluorid, das dreibasische Phosphat, das zweibasische Phosphit und viele andere entsprechend den Erdalkalisalzen wenig löslich und haben meist einen negativen Temperaturkoeffizienten. Es sind dies vielfach die Salze solcher Säuren, die wie die Fluorwasserstoffsäure und wohl auch die Phosphorsäure and phosphorige Säure ${ }^{2}$ ) polymere oder zum mindesten assoziierte Moleküle auch in wäBrigen Lösungen bilden.

Die Untersuchung der höchstbasischen Lithiumsalze der Tellursäure, der Antimonsäure und Perjodsäure ${ }^{3}$ ) scheinen diesen Zusammenhang zu bestätigen und zeigen, daB diese kristallisierten Verbindungen wie Kolloide aus konzentrierten Lithiumlaugen Lithiumbydroxyd stark adsorbieren.

Bei der Darstellung des kristallisierten Lithiumsilicats, $\mathrm{Li}_{2} \mathrm{SiO}_{3}$. $\mathrm{H}_{2} \mathrm{O}$, hat K. A. VesterberG ${ }^{4}$ ) Beobachtungen gemacht, die ähnliche Deutungen zulassen.

1) W. RzaLn, Inauguraldissertation (Manuskript), Berlin 1920.

2) Auce Italiener: „Über phosphorige Säure und ihre Salze“, Dissertation, Berlin 1917.

9) A. Rogenheim und G. JANDER, Kolloid-Zeitschr. 22 (1918), 1; G. J Kolloid-Zeitschr. 24 (1918), 4; A. Rosenheim u. E. Loewexthax, Kolloid-Zeitschr. 25 (1919), 53.

4) Z. anorg. u. allg. Chem. 110 (1920), 48. 
Diese Gesichtspunkte gaben die Veranlassung, eine Reihe von hochbasischen Lithiumsalzen schwacher anorganischer Säuren darzustellen und ihr Verhalten in Lösungen zu untersuchen in der Absicht, zu ermitteln, ob auch bei ihnen sich die Eigenschaften kolloider oder halbkolloider Stoffe zeigen würden.

\section{2-Lithium-1-Hydro-phosphit.}

Durch Zusatz von Lithiumcarbonat oder Lithiumhydroxyd zu einer Lösung von phospboriger Säure, bis Methylorange in der Farbe umschlug, erhielt AMAT $^{1}$ ) aus sirupöser Lösung das sehr leicht lösliche Lithium-2-Hydro-phosphit, $\mathrm{LiH}_{2} \mathrm{PO}_{3}$.

Dampft man eine Lösung ron $1 \mathrm{Mol} \mathrm{Li}_{2} \mathrm{CO}_{3}$ in $1 \mathrm{Mol} \mathrm{H}_{3} \mathrm{PO}_{3}$ Lösung auf dem Wasserbade ein, so scheidet sich in der Wärme als kristallinischer, aus feinen, vierseitigen Blättchen bestehender Niederschlag das 2-Lithium-1-Hydrophosphit ab. Die Analyse ${ }^{2}$ ) der Verbindung führte zu der Formel.

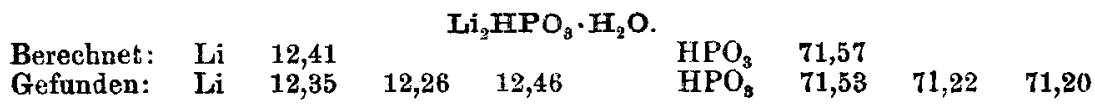

Die Löslichkeitsbestimmungen der Verbindung zeigten, wie schon bei der Darstellung beobachtet wurde, daB dieselbe einen negativen Temperaturkoeffizienten hat:

Löslichkeit von $\mathrm{Li}_{2} \mathrm{HPO}_{\mathrm{g}} \cdot \mathrm{H}_{2} \mathrm{O}$ in Wasser.

$\begin{array}{cccccccccc}t: & 0 & 25 & 30 & 35 & 40 & 45 & 51 & 61 & 98 \\ \mathrm{Li}_{2} \mathrm{HPO}_{\mathrm{B}} \text { in } 100 \mathrm{~g} \text { Lösung: } & 9,07 & \mathbf{7 , 4 7} & \mathbf{7 , 0 7} & \mathbf{6 , 8 2} & \mathbf{6 , 6 4} & \mathbf{6 , 2 9} & \mathbf{6 , 0 9} & \mathbf{5 , 7 5} & \mathbf{4 , 2 4}\end{array}$

Der Bodenkörper war innerhalb des ganzen untersuchten Gebietes das 1-Hydrat.

Diese geringe Löslichkeit unterscheidet sich außerordentlich von der des entsprechenden Natriumsalzes, $\mathrm{Na}_{2} \mathrm{HPO}_{3} \cdot 5,5 \mathrm{H}_{2} \mathrm{O}$, die A. ITALIENer bestimmt hat. ${ }^{3}$ )

Löslichkeit von $\mathrm{Na}_{2} \mathrm{HPO}_{8} \cdot 5,5 \mathrm{H}_{2} \mathrm{O}$ in Wasser (A. Italiener).

$\begin{array}{rcccccc}t: & 0 & 20 & 25 & 30 & 38 & 43 \\ \mathrm{Na}_{.2} \mathrm{HPO}_{3} \text { in } 100 \mathrm{~g} \text { Lösung: } & \mathbf{8 0 , 7 2} & \mathbf{8 1 , 0 6} & \mathbf{8 2 , 2 1} & 84,96 & \mathbf{9 1 , 9 4} & \mathbf{9 2 , 7}\end{array}$

Die Wasserstoffionenkonzentration einer äquivalent nor-

1) Ann. Chim. Phys. [6] 24 (1891), 308.

2) Nach A. IraLIEN Es (l. c.) lassen sich phosphorige Säure und Alkaliphosphite alkalimetrisch bzw. azidimetrisch scharf titrieren, und zwar erfolgt der Farbenumschlag bei Anwendung von Methylorange als Indikator bei der Bildung des einbasischen Alkali-2-Hydrophosphits bei Anwendung von $\alpha$-Naphtholphthaleins bei der Bildung des zweibasischen 2-Alkali-1-Hydrophosphits.

s) Dissert. (1. c.), S. 28. 
malen Lösung von $\mathrm{Li}_{2} \mathrm{HPO}_{3} \cdot \mathrm{H}_{2} \mathrm{O}$ bei $20^{\circ}$ wurde nach der Gaskettenmethode durch Bestimmung des Potentials der Wasserstoffelektrode in 1-n. $\mathrm{Li}_{2} \mathrm{HPO}_{3}$-Lösung gegen eine gesättigte Kalomelelektrode gemessen. Es ergab sich als Mittel der Messungen an drei Elektroden: die Wasserstoffzahl $p \cdot \mathrm{H}=8,14$, und mithin die Wasserstoffionenkonzentration $\left[\mathrm{H}^{\cdot}\right]=\mathbf{7 , 3 4} \cdot 10^{-\theta} \mathrm{g}$ im Liter bei $20^{\circ}$.

Dieser Wert steht in guter Übereinstimmung mit dem von A. ITALJENER ${ }^{2}$ ) nach der Indikatorenmethode von SöRENSEN für die Lösung von $\mathrm{Na}_{2} \mathrm{HPO}_{3}$ ermittelten Werte: $\left[\mathrm{H}^{\cdot}\right]=1 \cdot 10^{-9} \mathrm{~g}$ im Liter bei $20^{\circ}$.

Dies beweist, dab, wie zu erwarten ist, trotz der verschiedenen Löslichkeit der recht hohe Hydrolysengrad des Lithium- und Natriumsalzes der GröBenordnung nach gleich ist.

Aquivalentes Leitvermögen von $\mathrm{Li}_{2} \mathrm{HPO}_{8}$-Lösungen bei $25^{\circ}$.

$$
\begin{array}{ccccccc}
c: & 32 & 64 & 128 & 256 & 512 & 1024 \\
\lambda: & 68,1 & 72,6 & 76,4 & 82,1 & 86,0 & 102,4 \\
& \multicolumn{7}{r}{A=\lambda_{1024}=\lambda_{22}=34,3 .}
\end{array}
$$

\begin{tabular}{|c|c|c|c|c|c|c|}
\hline $\begin{array}{c}\mathrm{Li}_{3} \mathrm{HPO}_{\mathrm{z}} \cdot \\
\mathrm{H}_{2} \mathrm{O} \\
\text { in } \mathrm{g}\end{array}$ & $\begin{array}{c}\mathrm{Li}_{2} \mathrm{HPO}_{8} \\
\text { wasser- } \\
\text { frei } \\
\text { in } \mathrm{g}\end{array}$ & $\begin{array}{c}\text { Wasser } \\
\text { in } g\end{array}$ & $\begin{array}{l}\text { Gefrier- } \\
\text { punkts- } \\
\text { erniedri- } \\
\text { gung } \\
\text { in }\end{array}$ & $\begin{array}{c}\text { Molare } \\
\text { Konzen- } \\
\text { tration } \\
\text { im Liter }\end{array}$ & $\begin{array}{l}\text { Molare Ge- } \\
\text { frierpunkts- } \\
\text { erniedrigung } \\
\text { des Wassers } \\
\text { gefunden }\end{array}$ & $\begin{array}{c}\text { Vax'T } \\
\text { Horsscher } \\
\text { Faktor } \\
\text { J. }\end{array}$ \\
\hline 0,4014 & $0, \mathbf{3 3 6 8}$ & 19,243 & 0,820 & 0,1864 & 43,99 & 2,37 \\
\hline 0,4934 & 0,4141 & 19,26 & 0,890 & 0.229 & 38,88 & 2,09 \\
\hline 0,6689 & 0,5614 & 19,29 & 1,2830 & 0,3098 & 41,40 & 2,23 \\
\hline 0.9095 & 0,7533 & 19,33 & 1,7550 & 0,420 & 41,75 & 2,25 \\
\hline 1,1175 & 0,9380 & 19,36 & 2,208 & 0,516 & 42,80 & 2,30 \\
\hline 1,2445 & 1,0440 & 19,38 & 2,363 & 0,574 & 41,19 & 2,21 \\
\hline 1,4076 & 1,1810 & 19,41 & 2,710 & 0,648 & 41,82 & 2,25 \\
\hline
\end{tabular}

Gefrierpunktserniedrigung von $\mathrm{Li}_{2} \mathrm{HPO}_{3}$ - Lösung.

Aus diesen Werten lassen sich Schlüsse auf eine Assoziation des Phosphits etwa $\mathrm{zu}\left(\mathrm{Li}_{2} \mathrm{HPO}_{3}\right)_{2}$, wie sie die Messungen von A. ItaIIENer für die phosphorige Säure wahrscheinlich machen, nicht ziehen, sie stehen im Einklang mit den Werten, die für ein etwas hydrolytisch gespaltenes zweibasisches Salz zu erwarten sind.

Anzeichen für adsorptive Eigenschaften des $\mathrm{Li}_{2} \mathrm{HPO}_{3} \cdot \mathrm{H}_{2} \mathrm{O}$ wurden nicht gefunden. Auch aus konzentrierten Lösungen von $\mathrm{LiOH}$ schied sich das $\mathrm{Salz}$ rein aus, ohne $\mathrm{LiOH}$ zu adsorbieren.

t) Dissert., S. 14. 


\section{Lithiumsubphosphat, $\mathrm{Li}_{2} \mathrm{PO}_{3} \cdot 3,5 \mathrm{H}_{2} \mathrm{O}$.}

SaIzer ${ }^{1}$ ), sowie Rammelberg ${ }^{2}$ ) haben dies Salz als schwer löslichen Niederschlag durch Absättigung von Unterphosphorsäure mit Lithiumcarbonat erhalten. Übersättigt man eine wäbrige Lösung von Natriumbydrosubphosphat, $\mathrm{NaHPO}_{3} \cdot 3 \mathrm{H}_{2} \mathrm{O}$, mit Lithiumhydroxyd, so fällt die Verbindung in weißen, mikroskopischen, farrea " krautähnlichen Kristallen aus.

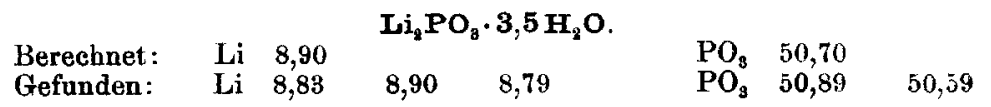

Bei Versuchen, die Löslichkeit dieses Salzes zu bestimmen, zeigte es sich, dab nach den üblichen Methoden die Absättigung auberordentlich langsam vor sich ging und dann nur trübe, auch bei mehrtägigem Absetzen sich nicht klärende Lösungen erhalten wurden, die scheinbar kolloidal, auch durch Filtration kaum zu reinigen waren. Die ausgeführten Bestimmungen konnten demnach zuverlässige Werte nicht ergeben und waren dieselben jedenfalls viel zu hoch. Es seien deshalb nur die folgenden Zahlen angeführt, die zeigen, daB die Verbindung sehr wenig löslich ist und wahrscheinlich auch einen negativen Temperaturkoeffizienten der Löslichkeit hat.

Bei $0^{\circ}$ enthielt eine $504 \mathrm{Stdn}$. abgesätt. Lös. in $100 \mathrm{~g} 0,1018 \mathrm{~g} \mathrm{Li}_{2} \mathrm{PO}_{3}$

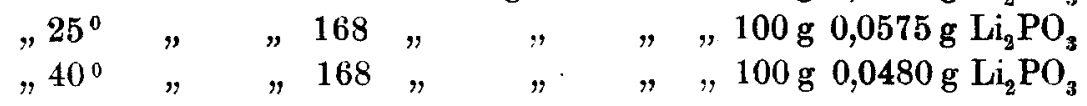

Es wurde daher versucht, eine genaue Bestimmung der Lơslichkeit aus dem Leitvermögen der gesättigten Lösungen nach der von BöTTGER $^{3}$ ) vielfach angewandten Methode $\mathrm{zu}$ berechnen. $\mathrm{Zu}$ diesem $Z$ wecke mußten die Wanderungsgeschwindigkeiten der Ionen des Salzes bekannt sein. Die des Lithiums ist nach KoHLRAUSCH und HoLBORN ${ }^{4}$ ) $U_{\mathrm{Li}}=33,4$ bei $18^{\circ}$ im reziproken Ohm und der Temperaturkoeftizient $=0,027$ pro Grad.

Um die Wanderungsgeschwindigkeit des $\left(\mathrm{PO}_{3}\right)^{\prime \prime}$-Anions zu ermitteln, wurde die Überführungszahl des $\mathrm{HPO}_{3}^{\prime}$-Anions in einer 0,1-n. Lösung von $\mathrm{NaHPO}_{3} \cdot 3 \mathrm{H}_{2} \mathrm{O}$ bei $16^{\circ}$ bestimmt. Verwendet wurde ein aus zwei U-Röhren zusammengesetzter Apparat

1) Ann. 194, 32.

2) Journ. prakt. Chem. [2] 45 (1892), 153.

3) Z. phys. Chem. 46 (1903), 521.

") „Das Leitvermögen der Elektrolyte“, II. Auflage, 1916, Tabelle 8 a. 
mit Platinelektroden, ähnlich der von $\mathrm{W} . \mathrm{BEIN}^{\mathrm{j}}$ ) angegebenen Vorrichtung. Eingeschaltet war ein Silbervoltameter; analysiert wurde jedesmal sowohl die Anoden-, wie die Kathoden- und die unveränderte Mittelschicht.

Drei Versuche von sehr verschiedener Zeitdauer ergaben für die Überführungszahl von $\left(\mathrm{HPO}_{3}\right)^{\prime}$ :

$$
\begin{aligned}
\text { I. } & 0,423 \text {, } \\
\text { II. } & 0,426 \text {, } \\
\text { III. } & 0,479 \text {. }
\end{aligned}
$$

Da der letzte Wert mit einem kleinen Versuchsfehler behaftet war, ist als Mittel 0,425 anzusehen.

Um hieraus mit Hilfe der Formel von HittorF:

$$
n=\frac{v}{u+v}
$$

die Wanderungsgeschwindigkeit $v$ des $\mathrm{HPO}_{3}$-Ions zu berechnen, ist die Wanderungsgeschwindigkeit $u$ von $\mathrm{Na}$ einzusetzen. Dieselbe bebeträgt im reziproken $\mathrm{Ohm}$ für $18^{\circ}$ nach KoHLRAUsch-HoLBORN $u=36,4$ für 0,1-n. Lösungen und bei Annahme des Temperaturkoeffizienten $0,027 u^{16^{\circ}}=34,4$. Hieraus ergibt sich:

$$
v^{160}=\frac{n \cdot u}{1-n}=\frac{0,425 \cdot 34,4}{0,575}=25,4
$$

und bei Annahme des 'Temperaturkoeffizienten $0,027 \quad v_{\mathrm{HPO}_{\mathbf{2}}^{\prime}}^{250^{\circ}}=\mathbf{3 1 , 6}$ in reziproken $\mathrm{Ohm}$.

Dieser Wert kann naturgemäß nur ein roher Näherungswert sein ${ }^{2}$; denn er ist unter der Voraussetzung erhalten, dab $\mathrm{NaHPO}_{3}$ nur in $\mathrm{Na}^{-}$- und $\mathrm{HPO}_{3}-$-Tonen dissoziiert, was nicht roll zutrifft, wie sich aus den unten verzeichneten Werten des äquivalenten Leitrermögens von $\mathrm{NaHPO}_{3}$-Lösungen ergibt. Immerhin findet er seine Bestätigung beim Vergleiche mit den Werten des äquivalenten Leitvermögens. Dasselbe wurde mit einem ganz reinen, mehrfach sorgfältig umkristallisierten Präparat von $\mathrm{NaHPO}_{3} \cdot 3 \mathrm{H}_{2} \mathrm{O}$ bestimmt, und es wurden folgende Zahlen erhalten, die mit den früher von A. RoseNHEIM und W. STADLER ${ }^{\text {? }}$ erhaltenen Werten verglichen seien.

1) Z. phys. Chem. 27 (1898), 1.

2) Die Wahrscheinlichkeit dieses Wertes wird immerhin beststigt durch die von Weasoheider für das Phosphation $\mathrm{H}_{2} \mathrm{PO}_{4}$ " ermittelte Wanderungsgeschwindigkeit $v_{\mathrm{H}_{2} \mathrm{PO}_{2}^{\prime \prime}}^{2 \tilde{s}^{\prime \prime}}=33,5$.

э) Ber. 39 (1906), 2839. 
Aquivalentes Leitvermögen von $\mathrm{NaHPO}_{8} \cdot 3 \mathrm{H}_{2} \mathrm{O}$ bei $25^{\circ}$.

$\begin{array}{rrrrrrrrr}\text { A: } & \mathbf{7 1 , 8} & \mathbf{7 8 , 8} & \mathbf{8 6 , 1} & \mathbf{9 2 , 2} & \mathbf{9 5 , 9} & \mathbf{9 8 , 1} & \mathbf{1 0 0 , 7} & \mathbf{1 0 1 , 6} \\ \lambda \text { (R. u. St.): } & & \mathbf{7 8 , 8} & \mathbf{8 1 , 6} & \mathbf{8 8 , 1} & \mathbf{9 4 , 5} & \mathbf{1 0 0 , 2} & \mathbf{1 0 5 , 9} & \mathbf{1 1 1 , 8}\end{array}$

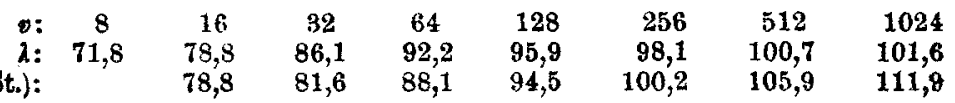

Aus den obigen Wanderungsgeschwindigkeiten für $\mathrm{Na}$ und $\mathrm{HPO}_{3}$ bei $25^{\circ}$ in 0,1-n. Lösung ergibt sich nach dem KoHLRAUschschen Gesetze, das streng allerdings nur für unendliche Verdünnung gilt, das äquivalente Leitvermögen für $\mathrm{NaHPO}_{3}$ in $0,1-\mathrm{n}$. Lösung $\lambda=u_{\mathrm{Na}}^{250}+v_{\mathrm{HPO}_{\mathrm{s}}}^{250}=43,3+31,6=74,9$, während oben für $v=8$ $\lambda=71,8$ und $v=16 \quad \lambda=78,8$ gefunden wurde.

$\mathrm{Um}$ nun aus der Wanderungsgeschwindigkeit von $\mathrm{HPO}_{3}{ }^{\prime}$ die des $\mathrm{PO}_{\mathrm{s}}{ }^{\prime \prime}$-Ions abzuleiten, wurde eine von WEGSCHEIDER ${ }^{1}$ ) aufgefundene Beziehung zwischen den Wanderungsgeschwindigkeiten ein- und zweiwertiger Ionen zweibasischer Säuren, die sich um $\mathrm{H}$. unterscheiden, verwendet. So beträgt dieses Verhältnis z. B. für $\mathrm{HPO}_{4}^{\prime \prime} \mathrm{zu} \mathrm{H}_{2} \mathrm{PO}_{4}^{\prime} 1,64$.

Nimmt man dasselbe Verhältnis für $\mathrm{PO}_{3}{ }^{\prime \prime} \mathrm{zu} \mathrm{HPO}_{3}$ an, so ist $v_{\mathrm{PO}_{z^{\prime \prime}}}^{2 \bar{o}^{\circ}}=51,8$ für 0,1-n. Lösung.

Für unendlich verdünnte Lösungen ist die Wanderungsgeschwindigkeit im allgemeinen $15-16 \%$ größer als für $0,1-n$. Lösungen. ${ }^{2}$ ) Es wiurde danach annähernd werden $v_{\mathrm{PO}_{\mathrm{a}^{\prime \prime}}}^{20^{\circ}}=60,8$. Da für unendlich verdünnte Lsöungen $u_{\mathrm{Li}}^{25^{0}}=39,7$ isti ${ }^{2}$ ), so wird das äquivalente Leitvermögen für $\mathrm{Li}_{2} \mathrm{PO}_{3}$ bei $25^{\circ}$ nach dem KOHLRAUsCHschen Gesetz:

sein.

$$
\lambda_{\infty}^{255^{\circ}}=u_{\mathrm{Li}}^{250^{\circ}}+v_{\mathrm{PO}_{3}}^{250^{\circ}}=39,7+60,8=100,5
$$

Zur Bestimmung des Leitvermögens der gesättigten $\mathrm{Li}_{8} \mathrm{PO}_{3}$ Lo̊sung wurde ein Lithiumsubphosphat verwendet, das durch Fällung von reinstem $\mathrm{NaHPO}_{3} \cdot 3 \mathrm{H}_{2} \mathrm{O}$ mit reinster Lithiumbydroxydlösung and erschöpfendem Auswaschen zuletzt mit "Leitfähigkeitswasser" $\left(k=3,2 \cdot 10^{-6}\right)$ dargestellt war. Die gesättigte Lösung wurde in dem aus Jenaer Glas hergestellten und gedämpften Leitfähigkeitsgefäb dargestellt. Im GefäB befand sich stets ein ÜberschuB des Bodenkörpers. Das Leitvermögen der Lösungen wurde nach mehrtägigem Schütteln gemessen und die Messungen nach stets erneutem Schütteln so lange wiederholt, bis Konstanz eingetreten war. Dann

1) Sitzungsber. d. Akad. d. Wissensch. Wien 101 (1902), 10; vgl. BörtGER, Z. phys. Ohem. 46 (1903), 593.

2) Vgl. Konlrausch, Hasbow, l. c., Tabelle 8 a. 
wurde die Lösung abgegossen und ein neuer AufguB hergestellt und -gemessen.

Die ersten Lösungen konnten noch geringe Mengen des leichter löslichen $\mathrm{Na}_{2} \mathrm{PO}_{3}$ enthalten. Es wurden folgende Werte erhalten:

Spezifisches Leitvermögen von gesättigten $\mathrm{Li}_{2} \mathbf{P O}_{8}$-Lösungen bei $25^{\circ}$.

$$
\begin{aligned}
& \text { AufguB 1 . . , . . . . . } k=\mathbf{5 , 1 1} \cdot 10^{-4} \\
& \text { "2....... . } k=3,09 \cdot 10^{-4}
\end{aligned}
$$

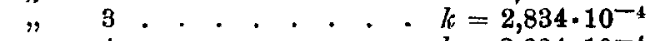

$$
\begin{aligned}
& \text { "4. . . . . . . . } k=2,694 \cdot 10^{-4} \\
& \text { " } 5 \text {. . . . . . . . } k=2,799 \cdot 10^{-4}
\end{aligned}
$$

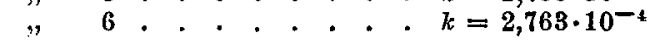

$$
\begin{aligned}
& \text { Mittel von 3-6. . . . . . } k=2,772 \cdot 10^{-4} \\
& \text { Leitfähigkeitswasser } \cdot \cdot \frac{k=0,032 \cdot 10^{-4}}{k=2,740 \cdot 10^{-4}}
\end{aligned}
$$

Hieraus ergibt sich die äquivalente Konzentration der Lösung:

$$
\eta=\frac{k}{\lambda_{\infty}}=\frac{2,74 \cdot 10^{-4}}{100,5}=2,73 \cdot 10^{-6} \mathrm{Mol} \frac{\mathrm{Li}_{2} \mathrm{PO}_{3}}{2} \text { im Liter. }
$$

Das Äquivalentgewicht von $\mathrm{Li}_{2} \mathrm{PO}_{3}$ beträgt 46,46. Mithin enthält bei $25^{\circ}$ ein Liter $\mathrm{Li}_{2} \mathrm{PO}_{3}$-Lösung 0,1267 $\mathrm{g} \mathrm{Li}_{2} \mathrm{PO}_{3}$.

Diese Zahl kann aus den oben angeführten Gründen, da die Wanderungsgeschwindigkeit des $\mathrm{PO}_{s}{ }^{\prime \prime}$-Ions nur annähernd zu bestimmen und mithin der Werte für $\lambda_{\infty}$ von $\mathrm{Li}_{8} \mathrm{PO}_{3}$ nur ein unterer Grenzwert ist, auf absolute Genauigkeit keinen Anspruch machen. Sie stellt aber, da wahrscheinlich sowohl die Wanderungsgeschwindigkeit wie $\lambda_{\infty}$ höher sind, einen oberen Grenzwert dar und beweist, daB die wahre Löslichkeit von $\mathrm{Li}_{2} \mathrm{PO}_{3}$ sicherlich viel geringer ist, als oben bei direkten Löslichkeitsbestimmungen gefunden wurde $\left(0,0575 \mathrm{~g} \mathrm{Li}_{2} \mathrm{PO}_{3}\right.$ in $100 \mathrm{~g}$ Lösung bei $25 \mathrm{~g}$. Ob die scheinbar kolloide Lösung als wirklich kolloidal anzusprechen ist, oder eine nichtkolloide Suspension ist, muB vorläufig dahingestellt bleiben.

\section{Lithiumphosphat, $\mathrm{Ii}_{3} \mathrm{PO}_{4}$.}

Das dreibasische Lithiumphosphat ist schon mehrfach untersucht worden und wird wegen seiner geringen Löslichkeit häufig zur analytischen Abscheidung des Lithiums empfohlen. Widerspruchsvoll sind die Angaben über den Wassergehalt des Salzes. Nach RAmmrasBerg ${ }^{1}$ ) hält es schwankende Mengen Wasser fest gebunden, nach W. MAXER ${ }^{2}$ ) entspricht es der Formel $2 \mathrm{Li}_{8} \mathrm{PO}_{4} \cdot \mathrm{H}_{2} \mathrm{O}$.

1) Pogg. Ann. 76 (1849), 261.

2) Ann. 98 (1856), 206. 
Durch Zusatz von Phosphorsäurelösung zu einem Überschuß von Lithiumhydroxydlösung erhält man das Salz als mikrokristallinischen, wenig löslichen Niederschlag. Die Analyse des gut Ausgewaschenen an der Luft bei ca. $16^{\circ}$ zur Gewichtskonstanz getrockneten Präparates führte zu der Formel;

\begin{tabular}{|c|c|c|c|c|c|c|c|c|}
\hline Berechnet: & $\mathbf{L i}$ & 13,70 & & $\mathrm{PO}_{4}$ & 62,58 & & $\mathrm{H}_{2} \mathrm{O}$ & 23,72 \\
\hline Gefunden: & $\mathbf{L i}$ & 13,50 & 13,36 & $\mathrm{PO}_{4}$ & 62,53 & 62,46 & $\mathrm{H}_{2} \mathrm{O}$ & 23,90 \\
\hline
\end{tabular}

Das Wasser wird aber entsprechend den Beobachtungen MAYRRs leicht abgegeben, und nach mehrtägigem Trocknen bei ca. $60^{\circ}$ erhält man Gewichtskonstanz bei Entstehung des $1 / 2$-Hydrates.

$$
\mathrm{Li}_{3} \mathrm{PO}_{4} \cdot 1 / 2 \mathrm{H}_{9} \mathrm{O}
$$

Berechnet: Li 16,67

Gefunden: $\quad$ Li $16,51 \quad 16,50$

$\mathrm{PO}_{4} \quad 76,10$

$\mathrm{H}_{2} \mathrm{O} \quad 7,23$

$\begin{array}{llllll}\mathrm{PO}_{4} & \mathbf{7 6}, 34 & \mathbf{7 6 , 1 8} & \mathrm{H}_{2} \mathrm{O} & \mathbf{7 , 3 0} & \mathbf{7 , 2 7}\end{array}$

Nach Rammersberg löst sich 1 Teil des Salzes in 833 Teilen Wasser, d. h. $0,120 \mathrm{~g}$ in $100 \mathrm{~g}$ Wasser, nach MaYer dagegen bei $15-18^{\circ} 1$ Teil in 2539 Teilen Wasser, d. h. $0,0394 \mathrm{~g}$ in $100 \mathrm{~g}$ Wasser.

Bei Versuchen, die Löslichkeit möglichst genau nach den üblichen Methoden zu bestimmen, wurden dieselben Beobachtungen wie bei dem Subphosphat gemacht: es warden nach mehrwöchentlicher Absättigung (14-21 'lage) bei konstanter Temperatur nur kolloidartige unfiltrierbare Suspensionen erhalten, deren analytische Untersuchung zu reproduzierbaren Werten nicht führten.

Es wurde deshalb ebenso wie beim Subphosphat die Löslichkeit aus der Bestimmung des spezifischen Leitvermögens der bei $25^{0}$ gesättigten Lösung ermittelt.

Spezifisches Leitvermögen der gesättigten $\mathrm{Li}_{8} \mathrm{PO}_{4}$-Lösung bei $25 "$.

$$
\begin{aligned}
& \text { AufguB 1 . . . . . . . . } k=20,1 \cdot 10^{-4}
\end{aligned}
$$

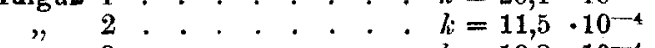

$$
\begin{aligned}
& \text {, } 3 . . . \text {. . . . . } k=10,8 \cdot 10^{-4} \\
& \text { " } \quad \begin{array}{l}
4 \\
5
\end{array} \quad \cdot \cdot \cdot \cdot \cdot \cdot \cdot \cdot k=9,51 \cdot 10^{-4} \\
& " \quad 6 \cdot \cdot \cdot \cdot \cdot \cdot \cdot \cdot k=9,43 \cdot 10^{-4} \\
& \text { " } 7.5 . \cdot . \cdot . \cdot . \cdot k=9,24 \cdot 10^{-4} \\
& \text { " 8. . . . . . . . } k=9,25 \cdot 10^{-4} \\
& \text { Mittel von 4-8 . . . . . . } k=9,40 \cdot 10^{-4}
\end{aligned}
$$

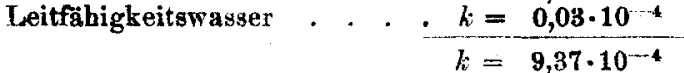


Die Wanderungsgeschwindigkeit des Li-Ions ist bei $25^{\circ}=39,7$, die des $\mathrm{PO}_{\text {" }}$-'Ions nach BöTTGER ${ }^{1}$ ) $=82,3$. Es ergibt sich hiermit die äquivalente Konzentration der Lösung:

$$
\eta=\frac{k}{\lambda_{\infty}}=\frac{9,37 \cdot 10^{-4}}{122}=7,688 \cdot 10^{-6} \mathrm{Mol} \frac{\mathrm{Li}_{3} \mathrm{PO}_{4}}{3} \text { im Liter. }
$$

Das Äquivalentgewicht von $\mathrm{Li}_{3} \mathrm{PO}_{4}$ beträgt 38,7 . Mithin enthält bei $25^{\circ}$ ein Liter $\mathrm{Li}_{3} \mathrm{PO}_{4}$-Lösung $0,297 \mathrm{~g} \mathrm{Li}_{3} \mathrm{PO}_{4}$. Die Löslichkeit ist hiernach höher als die des Subphosphats. Der orhaltene Wert steht dem von Mayer gefundenen am nächsten.

\section{Drei- and vierbasische Lithiumvanadinate.}

Während die sauren Vanadinate entsprechend den Salzen der anderen schwachen Metallsäuren, wie die Molybdänate usw., zu den Polysalzen komplizierter Zusammensetzung gehören, entsprechen die hochbasischen Reihen der Alkalivanadinate den Phosphaten in ihrer Zusammensetzung. Außer den dreibasischen "Alkaliorthovanadinaten" $\mathrm{R}_{3} \mathrm{VO}_{4} \cdot \mathrm{xH}_{2} \mathrm{O}$ existieren nach DITrE ${ }^{2}$ ) noch vierbasische Alkalivaradinate, die durch Eintragen von Vanadiumpentoxyd in überschüssige Alkalilaugen entstehen sollen. Er beschreibt hier - allerdings ohne jede analytischen Belege - die Verbindingen:

$$
\begin{gathered}
4 \mathrm{~K}_{2} \mathrm{O} \cdot \mathrm{V}_{2} \mathrm{O}_{5} \cdot 20 \mathrm{H}_{2} \mathrm{O}-4 \mathrm{Na}_{2} \mathrm{O} \cdot \mathrm{V}_{2} \mathrm{O}_{5} \cdot 30 \mathrm{H}_{2} \mathrm{O}-4 \mathrm{Li}_{2} \mathrm{O} \cdot \mathrm{V}_{2} \mathrm{O}_{5} \cdot \mathrm{H}_{2} \mathrm{O}- \\
4 \mathrm{Li}_{2} \mathrm{O} \cdot \mathrm{V}_{2} \mathrm{O}_{\xi} \cdot 14 \mathrm{H}_{2} \mathrm{O} .
\end{gathered}
$$

Das 1-Hydrat des Lithiumsalzes soll sich beim Eintragen einer mit $\mathrm{V}_{2} \mathrm{O}_{5}$ abgesättigten Lösung von $\mathrm{Li}_{2} \mathrm{CO}_{3}$ in eine heiße, gesättigte Lithiumhydroxydlösung als weiBer Niederschlag, der aus durchsichtigen mikroskopischen Kristallen besteht, abscheiden. Das 14-Hydrat soll durch Umwandlung des 1-Hydrats beim Stehen unter der Lauge sich bilden und aus durchsichtigen, gut ausgebildeten Rhomboedern bestehen.

Ein Hydrat des lithiumorthoranadinats, $3 \mathrm{Li}_{2} \mathrm{O} \cdot \mathrm{V}_{2} \mathrm{O}_{5} \cdot 6 \mathrm{H}_{2} \mathrm{O}$, soll ebenfalls nach Ditre aus den sirupösen Laugen des Lithiumpyrovanadinats, $\mathrm{Li}_{4} \mathrm{~V}_{2} \mathrm{O}_{7} \cdot 6 \mathrm{H}_{8} \mathrm{O}$, als schwer von den Beimengungen zu trennende Kristallmasse sich abscheiden. Auch hier fehlen alle analytischen Daten.

Trägt man in einen großen Überschuß einer gesättigten Lithiumbydroxydlösung (4-4,5-n.) unter Umrühren bei Zimmertemperatur

2) Z. phys. Chem. 46 (1903), 596.

7) Compt. rend. 104 (1887), 902, 1061, 1163. 
Vanadiumpentoxyd ein, so geht dies klar in Lösung. Erhitzt man diese Lösung, so bildet sich sofort ein reichlicher Niederschlag, der aus mikroskopischen Sphärolithen besteht. Er wurde aus der heiBen Lösung schnell abgesaugt und zuerst auf dem Filter, dann auf Ton ohne besonderes Auswaschen trocken gepreBt.

Die Analyse ${ }^{1}$ ) dieser Verbindung, die mehrfach dargestellt wurde, führte zu der Formel:

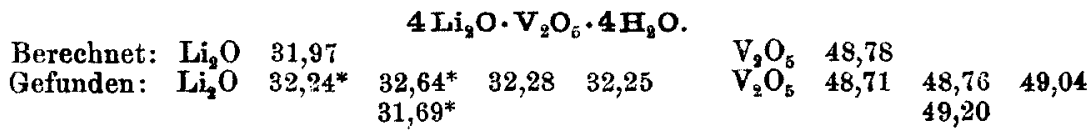

Diese Verbindung ist nach Entstehung und nach Aussehen identisch mit dem von DrrTw beschriebenen 1-Hydrat, unterscheidet sich aber in der analytischen Zusammensetzung grundlegend davon. Sie wird nur erhalten, wenn ein sehr groBer ÜberschuB ron konzentrierter Lithiumhydroxydlösung anwesend ist. Trägt man zu viel Vanadiumpentoxyd in die Lauge ein, so bilden sich möglicherweise Gemische des vierbasischen Salzes mit dem später zu behandelnden ihm äuBerlich ganz gleichen $3 \mathrm{Li}_{2} \mathrm{O} \cdot \mathrm{V}_{2} \mathrm{O}_{5} \cdot 2 \mathrm{H}_{2} \mathrm{O}$. So wurden bei verschiedenen Darstellungen folgende Werte erhalten:

\begin{tabular}{|c|c|c|c|c|}
\hline \multicolumn{2}{|c|}{ Berechnet: } & \multicolumn{3}{|c|}{ Gefunden: } \\
\hline $\begin{array}{c}4 \mathrm{Li}_{2} \mathrm{O} \cdot \mathrm{V}_{2} \mathrm{O}_{5} \cdot 4 \mathrm{H}_{2} \mathrm{O} \\
\mathrm{Li}_{2} \mathrm{O} \quad 31,97 \\
\mathrm{~V}_{2} \mathrm{O}_{5} \\
\mathbf{4 8 , 7 8}\end{array}$ & $\begin{array}{c}3 \mathrm{Li}_{2} \mathrm{O} \cdot \mathrm{V}_{3} \mathrm{O}_{5} \cdot 2 \mathrm{H}_{2} \mathrm{O} \\
29,17 \\
59,13\end{array}$ & $\begin{array}{c}\text { I. } \\
\mathbf{3 2 , 9 0} \\
55,34\end{array}$ & $\begin{array}{l}\text { Ir. } \\
32,73 \\
56,27\end{array}$ & $\begin{array}{l}\text { III. } \\
32,96 \\
54,82\end{array}$ \\
\hline
\end{tabular}

Immerhin wäre es auch möglich, daß, ähnlich wie bei dem Lithiumtellurat, Lithiumperjodat usw. das vierbasische Lithiumvanadinat nur durch Adsorption aus dem dreibasischen Salze entsteht. Die versuchte Durchführung von Adsorptionsversuchen scheiterte hier an der beträchtlichen Löslichkeit des Lithiumvanadinats bei gewöhnlicher Temperatur und dem dabei eintretenden komplizierten Gleichgewicht.

Die Abscheidung des Salzes $4 \mathrm{Li}_{2} \mathrm{O} \cdot \mathrm{V}_{2} \mathrm{O}_{5} \cdot 4 \mathrm{H}_{2} \mathrm{O}$ aus der Lösung bei höherer Temperatur sprach dafür, daB dasselbe einen negativen

1) Vanadiumpentoxyd wurde nach Reduktion mit $\mathrm{SO}_{2}$ in schwefelsaurer Lösung zu $\mathrm{V}_{2} \mathrm{O}_{4}$ maBanalytisch mit Permanganat bestimmt. Zur Lithiumbestimmung wurde teilweise eine neue azidimetrische, später zu veröffentlichende Methode verwandt, nach der Vanadinate bei Anwendung von $\alpha$-Naphtholphthalein als Indikator bei der Bildung von Metavanadinaten $\mathrm{RVO}_{3}$ umschlagen, teilweise wurde nach Entfernung der $\mathrm{V}_{2} \mathrm{O}_{5}$ mit Queeksilber(I.)nitrat oder Barythydrat, $\mathrm{Li}_{2} \mathrm{SO}_{4}$, gravimetrisch bestimmt. Die azidimetrisch ermittelten Werte sind durch * gekennzeichnet. 
Temperaturkoeffizienten der Löslichkeit besitzen müsse. Eine größere Rujihe von Löslichkeitsbestimmungen bei $0^{\circ}$ ergaben nun keine übereinstimmenden Werte, und es zeigte sich, daB bei dieser Temperatur der Bodenkörper sich allmählich veränderte und in die ron DrTTE als das Salz $4 \mathrm{Li}_{2} \mathrm{O} \cdot \nabla_{2} \mathrm{O}_{5} \cdot 14 \mathrm{H}_{3} \mathrm{O}$ bezeichneten schönen, durchsichtigen rhomboedrischen Nadeln überging. Hatte sich diese Umwandlung vollzogen, so enthielten bei $0^{\circ} 100 \mathrm{~g}$ der Lösung $2,18 \mathrm{~g}$ feste Substanzen.

Dasselbe Salz bildete sich naturgemäB in ganz reinem Zustande, wonn eine bei $0^{0}$ mit $\mathrm{V}_{2} \mathrm{O}_{5}$ teilweise abgesättigte Lithiumhydroxydlösang einige Zeit bei $0^{\circ}$ stand. Die schnell abgesaugte und auf Ton getrocknete Verbindung erwies sich als das 18-Hydrat des dreibasischen Lithiumvanadinats.

$$
3 \mathrm{Li}_{4} \mathrm{O} \cdot \nabla_{2} \mathrm{O}_{5} \cdot 18 \mathrm{H}_{2} \mathrm{O}=\mathrm{Li}_{8} \nabla \mathrm{O}_{4} \cdot 9 \mathrm{H}_{2} \mathrm{O}
$$

Borechnet: $\mathrm{Li}, \mathrm{O} \quad 15,03$

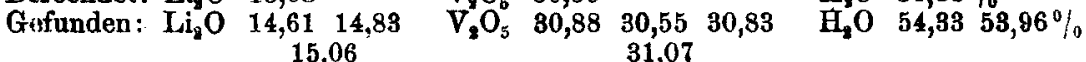

$\mathrm{V}_{9} \mathrm{O}_{6} \quad 30,59 \quad \mathrm{H}, \mathrm{O} \quad 54,38 \%$ 15,06 31,07

Dieses Salz ist unzweifelhaft identisch mit Drwass angeblichem $4 \mathrm{Li}_{2} \mathrm{O} \cdot \mathrm{V}_{2} \mathrm{O}_{5} \cdot 14 \mathrm{H}_{2} \mathrm{O}$ und, da es aus den Lösungen von $4 \mathrm{LiO} \cdot \mathrm{V}_{2} \mathrm{O}_{5}$. $4 . \mathrm{H}_{2} \mathrm{O}$ auskristallisiert, so ist dieses, wie auch zu erwarten war, offenbar hydrolytisch gespalten.

Beim Liegen an der Luft verwittert $\mathrm{Li}_{3} \nabla \mathrm{OO}_{4} \cdot 9 \mathrm{H}_{2} \mathrm{O}$ außerordentlich schnell, ein Zeichen, daB es wohl bei verhältnismäBig niedriger Timperatur in ein niedrigeres Hydrat sich umwandelt.

Thermometrische Bestimmungen eines Umwandlungspunktes ergaben, daB $\mathrm{Li}_{3} \mathrm{VO}_{4} \cdot 9 \mathrm{H}_{2} \mathrm{O}$ bei ca. $33^{\circ}$ zusammenzusintern beginnt, daB bei ca. $38^{\circ}$ eine klare wäBrige Lösung entsteht, die über einem feinpulverigen, aus mikroskopischen Sphärolithen bestehenden Bodenkörper sich befindet. Dieser wurde schnell abgesaugt und getrocknet; er gleicht äuBerlich vollständig dem $4 \mathrm{Li}_{2} \mathrm{O} \cdot \mathrm{V}_{2} \mathrm{O}_{5} \cdot 4 \mathrm{H}_{2} \mathrm{O}$.

Die Analyse der Verbindung fuhrte zu der Formel:

$$
3 \mathrm{Li}_{2} \mathrm{O} \cdot \mathrm{V}_{2} \mathrm{O}_{5} \cdot \mathbf{2} \mathrm{H}_{8} \mathrm{O} \text {. }
$$

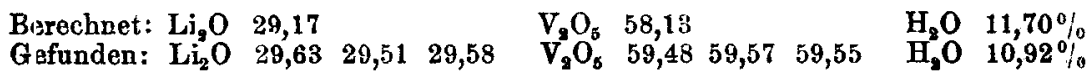

Dieselbe Verbindung erhält man, wenn man $\mathrm{Li}_{9} \mathrm{VO}_{4} \cdot 9 \mathrm{H}_{8} \mathrm{O}$ direkt entwässert. Entwässerungsreihen bei 35,78 und $100^{\circ}$ ergaben, daB $8 \mathrm{Mol}$ Wasser entweichen und $\mathrm{Li}_{3} \nabla \mathrm{O}_{4} \cdot \mathrm{H}_{2} \mathrm{O}$ zurückbleibt. Dieses 1.-Hydrat verliert das $1 \mathrm{Mol} \mathrm{H}_{2} \mathrm{O}$ erst beim Glühen. Bestätigt wurden diese Beobachtungen durch Löslichkeitsbestimmungen der Hydrate von $\mathrm{Li}_{3} \mathrm{VO}_{4}$. 
Bei niedrigen Temperaturen zwischen 0 und $35^{\circ}$, bei denen im System: $\mathrm{Li}_{3} \mathrm{VO}_{4}-\mathrm{H}_{2} \mathrm{O}$ das 9-Hydrat den Bodenkörper bildete, wurden klare Lösungen erhalten, deren Analyse zu eindeutigen Werten führte. Der Temperaturkoeffizient der Löslichkeit ist positiv. Oberhalb dieser Temperatur beginnt die Umwandlung des Bodenkörpers in das 1-Hydrat und nunmehr wurden nur trübe kolloidartige Lösungen erhalten, deren Analysen trotz sehr vielfacher Wiederholungen zu dauernd schwankenden Werten führten. Die Ursache dieser Erscheinung konnte nicht ermittelt werden; möglicherweise besteht der Bodenkörper aus einem Gemisch von $3 \mathrm{Li}_{2} \mathrm{O}$. $\mathrm{V}_{2} \mathrm{O}_{5} \cdot 2 \mathrm{H}_{2} \mathrm{O}$ und $4 \mathrm{Li}_{2} \mathrm{O} \cdot \mathrm{V}_{2} \mathrm{O}_{5} \cdot 4 \mathrm{H}_{2} \mathrm{O}$ oder das erstere $\mathrm{Salz}$ adsorbiert aus der Lösung das infolge von Hydrolyse entstandene LiOH. Sicher ist es aber, dab innerbalb dieses Temperaturgebietes der Temperaturkoeffizient der Löslicbkeit negativ ist.

Bei der Löslichkeitsbestimmung wurde der Gehalt der Lösungen titrimetrisch ermittelt und zugleich durch Eindampfen gewogener Lösungsmengen und Bestimmung des Glührückstandes kontrolliert. Die Bodenkörper wurden in den meisten Fällen analysiert.

Löslichkeit von $\mathrm{Li}_{3} \mathrm{VO}_{4} \cdot \mathrm{XH}_{2} \mathrm{O}$ in Wasser.

\begin{tabular}{|c|c|c|c|c|c|}
\hline$t^{0}$ & 0 & 20,8 & 28,6 & 30,2 & 35,2 \\
\hline $\begin{array}{r}\left.100 \mathrm{~g} I_{\text {Iösung enthalten }} \mathrm{Li}_{3} \mathrm{VO}_{4} \mathrm{~g}^{1}\right) \\
\text { Bodenkörper }\end{array}$ & 2,40 & $\begin{array}{l}4,60 \\
\mathrm{Li}_{8} \mathrm{VO}_{4}\end{array}$ & $\begin{aligned} 5,25 \\
9 \mathrm{H}, \mathrm{O}\end{aligned}$ & 5,91 & 6,25 \\
\hline g Lösung enthalten $\mathrm{Li}_{3} \mathrm{VO}_{4} \mathrm{~g}^{t^{0}}$ & $\begin{array}{l}38,4 \\
5,09\end{array}$ & $\begin{array}{l}40,0 \\
4,20 \\
\mathrm{Li}_{3} \mathrm{VO}_{4}\end{array}$ & $\begin{array}{l}45,0 \\
3,70 \\
\mathrm{H}_{z} \stackrel{0}{0}\end{array}$ & $\begin{array}{l}50 \\
2,80\end{array}$ & $\begin{array}{l}60 \\
2,60\end{array}$ \\
\hline
\end{tabular}

Während hiernach das 9-Hydrat wahre Lösungen ergibt, scheinen die des 1-Hydrats kolloidartigen Charakter zu haben.

Nach diesen Ergebnissen scheidet sich also aus Lösungen von Vanadiumpentoxyd in überschüssiger Lithiumlauge bei niedriger Temperatur bis ca. $35^{\circ}$ das 9 -Hydrat $\mathrm{Li}_{3} \mathrm{VO}_{4} \cdot 9 \mathrm{H}_{2} \mathrm{O}$ aus, das oberhalb dieser Temperatur in das 1-Hydrat $\mathrm{Li}_{3} \mathrm{VO}_{4} \cdot \mathrm{H}_{2} \mathrm{O}$ sich um. wandelt. Statt des letzteren entstehen aus den Lösungen bei Anwesenheit eines großen Überschusses von Lithiumhydroxyd Bodenkörper, die änBerlich dem $\mathrm{Li}_{3} \nabla \mathrm{VO}_{4} \cdot \mathrm{H}_{2} \mathrm{O}$ vollständig gleichen, aber in ihrer Zusammensetzung dem vierbasischen Salze $4 \mathrm{Li}_{2} \mathrm{O} \cdot \mathrm{V}_{2} \mathrm{O}_{5} \cdot 4 \mathrm{H}_{2} \mathrm{O}$ entsprechen oder nahe kommen. Ob hier eine chemische Verbindung vorliegt oder Adsorption eingetreten ist, bleibt noch unentschieden.

1) Mittel aus je 4 bis 6 Bestimmungen, die wäbrend der Anwesenheit des 9-Hydrats als Bodenkörper in guter Ubereinstimmung waren, für höhere Temperaturen aber stark differierten. 


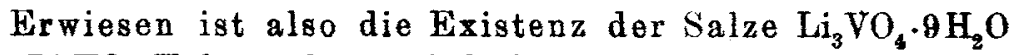
und $\mathrm{Li}_{3} \mathrm{VO}_{4} \cdot \mathrm{H}_{2} \mathrm{O}$, wahrscheinlich aber unentschieden die des Salzes $4 \mathrm{Li}_{2} \mathrm{O} \cdot \nabla_{2} \mathrm{O}_{5} \cdot 4 \mathrm{H}_{2} \mathrm{O}$. Es existieren dagegen nicht die ron DitTe beschriebenen Salze $4 \mathrm{Li}_{8} \mathrm{O} \cdot \mathrm{V}_{2} \mathrm{O}_{5} \cdot \mathrm{H}_{2} \mathrm{O}$ und $4 \mathrm{Li}_{2} \mathrm{O} \cdot \mathrm{V}_{2} \mathrm{O}_{5} \cdot 14 \mathrm{H}_{2} \mathrm{O}$ - letzteres ist identisch mit $\mathrm{Li}_{3} \mathrm{VO}_{4} \cdot 9 \mathrm{H}_{2} \mathrm{O}$ - sowie $\mathrm{Li}_{3} \mathrm{VO}_{4} \cdot 6 \mathrm{H}_{2} \mathrm{O}$.

\section{v. Lithiummolybdänat und Lithiumwolframat.}

Das höchstbasische Lithiummolybdänat hat RAMMELSBERG ${ }^{1}$ ) durch Kochen einer Suspension von $1 \mathrm{Mol} \mathrm{Li}_{2} \mathrm{CO}_{3}$ und $1 \mathrm{Mol} \mathrm{MoO}_{3}$ in dünnen, prismatischen Nadeln erhalten. Er findet für dasselbe die Formel $5 \mathrm{Li}_{2} \mathrm{MoO}_{4} \cdot 2 \mathrm{H}_{2} \mathrm{O}$, die WEMPE ${ }^{2}$ ) später bestätigte, während F. LPHRaIM and M. BRAND ${ }^{3}$ die Verbindung für wasserfrei erklärten. Das böchstbasische Lithiumwolframat ist bisher nur von E. F. ANTHoN ${ }^{4}$ ) beschrieben worden. Es soll aus Lösungen einer Schmelze von $1 \mathrm{Mol} \mathrm{Li}_{2} \mathrm{CO}_{3}$ und $1 \mathrm{Mol} \mathrm{WO}_{3}$ auskristallisieren; analytische Angabın fehlen vollständig.

Aus einer Lösung, die durch Kochen einer Suspension von $1 \mathrm{LiCO}_{3}$ und $1 \mathrm{MoO}_{3}$ erhalten war, kristallisierten beim Eindampfen lange fächerförmig angeordnete Kristallnadeln aus, die unter dem Polarisationsmikroskop starke Lichtbrechung zeigten. Dieses Lithiummolybdänat reagierte in wäBriger Lösung neutral. Ganz ebenso wurde Lithiumwolframat dargestellt, das offenbar noch leichter löslich, als das Molybdänat als feinkörniger, aus mikroskopischen Kristallnadeln bestehender Niederschlag sich abschied. Die Lösung dieses Salzes reagiert alkalisch.

Die Analysen der zentrifugierten lufttrockenen Präparate führte zu folgenden Ergebnissen:

\begin{tabular}{|c|c|c|c|c|c|c|c|c|}
\hline \multirow[b]{2}{*}{$\begin{array}{l}\text { Berechnet: } \\
\text { Gefunden: }\end{array}$} & \multicolumn{6}{|c|}{$4 \mathrm{Li}_{1} \mathrm{MoO}_{4} \cdot 3 \mathrm{H}_{2} \mathrm{O}}$. & \multirow{2}{*}{\multicolumn{2}{|c|}{$\begin{array}{ll}\mathrm{H}_{2} \mathrm{O} & 7,20 \% \\
\mathrm{H}_{8} \mathrm{O} & 7,67 \% \\
7,20 \%\end{array}$}} \\
\hline & $\begin{array}{l}\mathrm{Li}_{2} \mathrm{O} \\
\mathrm{Li}_{3} \mathrm{O}\end{array}$ & $\begin{array}{l}15,95 \\
16,09\end{array}$ & 16,28 & $\mathrm{MoO}_{3}$ & $\begin{array}{l}76,85 \\
78,78\end{array}$ & 76,41 & & \\
\hline \multicolumn{9}{|c|}{$4 \mathrm{Li}_{2} \mathrm{WO}_{4} \cdot 3 \mathrm{H}_{2} \mathrm{O}$} \\
\hline $\begin{array}{l}\text { Bereahnet: } \\
\text { Gefunden: }\end{array}$ & $\mathbf{L i}_{2} \mathbf{O}$ & $\begin{array}{l}10,85 \\
11,04\end{array}$ & 11,16 & $\mathrm{WO}_{3} 8$ & 4,33 & 1,37 & $\mathrm{H}_{2}^{2} \mathrm{O}$ & $4,90 \quad 4,97 \%$ \\
\hline
\end{tabular}

Die Analogie der beiden Formeln spricht bei diesen vielfach isomorphen Verbindungsreihen für die Richtigkeit des Befundes.

\footnotetext{
1) Pogg. Ann. 128, 311.

2) Z. anorg. Chem. 78 (1912), 298.

3) Z. anorg. Chem. 64 (1909), 258.

4) J. prakt. Chem. 8 (1836), 405.
} 
Für die ron RAMMELSBERG und WeMPE angegebene Zusammensetzung des Lithiummolybdänats $5 \mathrm{Li}_{2} \mathrm{MoO}_{4} \cdot 2 \mathrm{H}_{2} \mathrm{O}$ sind folgende Werte berechnet: $\mathrm{Li}_{2} \mathrm{O} \quad 16,56, \mathrm{MoO}_{3} 79,47, \mathrm{H}_{2} \mathrm{O} 3,97$.

Höher basische Salze waren hỉer nicht zu isolieren. Beide Verbindungen lösen sich sehr leicht in Lithiumhydroxydlösungen und geben beim Einengen derselben dann zähe, nicht kristallisierende Sirupe. Zum Unterschied von den meisten anderen neutralen Lithiumsalzen schwacher Säuren sind diese Salze recht leicht löslich.

Die Löslichkeitsbestimmungen des Lithiummolybdänats wurden dadurch erschwert, daB kolloidartige, nicht vollständig zu klärende Lösungen orhalten wurden. Die erhaltenen Werte sind daher sicherlich etwas zu hoch.

Löslichkeit von $4 \mathrm{Ii}_{2} \mathrm{MOO}_{4} \cdot 3 \mathrm{H}_{2} \mathrm{O}$ in Wasser.

$100 \mathrm{~g}$ Lösung enthalten $\mathrm{Li}_{9} \mathrm{MoO}_{4}$ in $\begin{array}{cccccc}t^{0}: & 0 & 25 & 30 & 40 & 98 \\ & \mathbf{4}:, 24 & 44,81 & 44,26 & 43,84 & \mathbf{4 2 , 5 0}\end{array}$

Die Löslichkeit hat auch hier einen negativen Temperaturkoeffizienten. Auffällig ist die außerordentlich geringe Abhängigkeit von der Temperaturänderung. Nach WEMPE enthält bei $20^{\circ}$ eine gesättigte Lösung des Salzes von der Dichte $1,44 \quad 46,30 \%$ $\mathrm{Li}_{2} \mathrm{MoO}_{4} \cdot 2 \mathrm{H}_{2} \mathrm{O}$. Dies entspricht $44,30 \mathrm{~g}$ wasserfreiem $\mathrm{Li}_{2} \mathrm{MoO}_{4}$ in $100 \mathrm{~g}$ Lösung.

Bei Versuchen, die Löslichkeit des Lithiumwolframats zu bestimmen, zeigte es sich, daB das stark hydrolytisch gehaltene $4 \mathrm{LiWO}$. $3 \mathrm{H}_{2} \mathrm{O}$ durch die Luftkohlensäure schnell weiter gespalten wurde. Der Bodenkörper enthielt dann ein Gemisch ron $\mathrm{Li}_{2} \mathrm{CO}_{3}$ und $\mathrm{WO}_{3}$.

\section{Lithiumborat.}

Aus einer Lösung von Borsäure in überschüssiger konzentrierter Lithiumlange kristallisiert in großen hexagonalen Platten das schon von Reischis ${ }^{1}$ ), sowie Le ChateureR ${ }^{2}$ ) erhaltene Lithiummetaborat8-Hydrat aus.

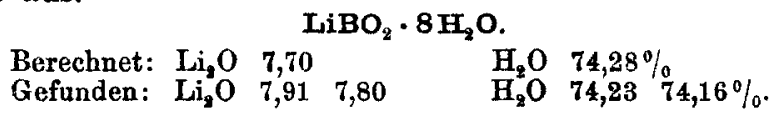

Ein lithiumreicheres Salz ist auch aus konzentriertester Lithiumlauge nicht $\mathrm{zu}$ erhalten.

Konstitutionswasserbestimmungen des Salzes ergaben wieder in Übereinstimmung mit REISCHLE, daB bis $110^{\circ} 65,3 \% \mathrm{H}_{2} \mathrm{O}$ entsprechend

1) Z. anorg. Chem. 4 (1893), 169.

2) Compt. rend. 124 (1897), 1091. 
$7 \mathrm{Mol} \mathrm{H}_{2} \mathrm{O}$ entweichen und $1 \mathrm{Mol}$ fest gebunden ist. Le Ceatedier folgert daraus und aus der hohen Gefrierpunktserniedrigung der Lösungen des Salzes, daB dieses als ein ,saures Orthoborat" $\mathrm{LiH}_{2} \mathrm{BO}_{3}$. $7 \mathrm{H}_{2} \mathrm{O} \mathrm{zu}$ betrachten sei. Die Ergebnisse der folgenden Tabelle bestätigen diese Annahme nicht.

Gefrierpunktserniedrigung wäbriger Lösungen von $\mathrm{LiBO}_{2} \cdot 8 \mathrm{H}_{2} \mathrm{O}$.

\begin{tabular}{c|c|c|c|c|c}
\hline $\begin{array}{c}\mathrm{LiBO}_{\mathbf{2}} \cdot 8 \mathrm{H}_{\mathbf{2}} \mathrm{O} \\
\text { in } \mathrm{g}\end{array}$ & $\begin{array}{c}\text { LiBO, } \\
\text { wasserfrei } \\
\text { in } \mathrm{g}\end{array}$ & $\begin{array}{c}\text { Wasser } \\
\text { in } \mathrm{g}\end{array}$ & $\begin{array}{c}\text { Gefrier- } \\
\text { punktsernie- } \\
\text { drigung in g }\end{array}$ & $\begin{array}{c}\text { Molare Ge- } \\
\text { frierpunkts- } \\
\text { erniedrigung } \\
\text { gefunden }\end{array}$ & $\begin{array}{c}\text { Dissoziations- } \\
\text { grade } \\
\text { J. }\end{array}$ \\
\hline \hline 0,0716 & 0,0184 & 24,90 & 0,051 & 34,42 & 1,86 \\
0,1539 & 0,0396 & $\mathbf{2 4 , 9 6}$ & $\mathbf{0 , 1 4 0}$ & $\mathbf{4 4 , 0 8}$ & $\mathbf{2 , 3 8}$ \\
0,2397 & 0,0617 & $\mathbf{2 5 , 0 3}$ & $\mathbf{0 , 2 1 2}$ & $\mathbf{4 2 , 9 5}$ & 2,32 \\
0,3523 & 0,0907 & 25,11 & 0,308 & $\mathbf{4 2 , 6 3}$ & $\mathbf{2 , 2 6}$ \\
0,5255 & 0,1852 & $\mathbf{2 5 , 2 4}$ & $\mathbf{0 , 4 1 1}$ & 38,32 & $\mathbf{2 , 0 7}$ \\
$\mathbf{0 , 6 8 2 3}$ & $\mathbf{0 , 1 7 5 6}$ & $\mathbf{2 5 , 3 6}$ & $\mathbf{0 , 5 1 8}$ & $\mathbf{3 7 , 3 6}$ & $\mathbf{2 , 0 4}$
\end{tabular}

Die Gefrierpunktserniedrigung ist besonders für die konzentrierteren Lösungen eine für einen binären Elektrolyten durchaus norraale.

Die für die Löslichkeit des Salzes gefundenen Werte stimmen dagegen im wesentlichen mit den Resultaten Le Chateliers überein.

Löslichkeit von $\mathrm{LiBO}_{2} \cdot 8 \mathrm{H}_{2} \mathrm{O}$ in Wagser.

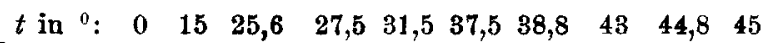

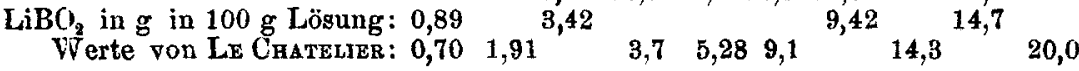

Bei $47^{\circ}$ schmilzt das Salz in seinem Kristallwasser. Auffällig ist das starke Ansteigen der Löslichkeit des Salzes bei höherer Temperatur rerglichen mit der geringen Zunahme bei niedrigeren Temperaturen; jedoch zeigte sich, daß innerhalb des ganzen untersuchten Temperaturgebietes der Bodenkörper unverändert blieb. Die von Le Chateidier für $45^{\circ}$ gefundene Löslichkeit scheint wesentlich zu hoch zu sein.

\section{Lithiumcarbonat.}

Nach den Bestimmungen ron BEWAD ${ }^{1}$ ) zeigt die Löslichkeit des Lithiumcarbonats einen negativen Temperaturkoeffizienten.

Iöslichkeit von $\mathrm{Li}_{2} \mathrm{CO}_{3}$ in Wasser (Bowad).

$\begin{array}{rcccccc}t^{0}: & 0 & 10 & 20 & 50 & 75 & 100 \\ \mathrm{Li}_{2} \mathrm{CO}_{\mathrm{g}} \text { in } \mathrm{g} \text { in } 100 \mathrm{~g} \text { Lösung: } & 1,54 & 1,44 & 1,33 & 1,18 & 0,89 & 0,73\end{array}$

1) Journ. russ. phys. chem. Ges. 16 (1884), 591.

z. anorg. u. allg. Ghem. Bd. 120. 
Es ist bekannt, daB wäBrige Lösungen von Lithiumcarbonat bei höherer Temperatur Kohlensäure verlieren und es muB dabei ein Gleichgewicht von $\mathrm{LiOH}: \mathrm{Li}_{2} \mathrm{CO}_{3}$ sich einstellen, das noch nicht untersucht ist. Wenn diese Beobachtungen richtig sind, können die in obiger Tabelle angegebenen Werte wenigstens für die höheren Temperaturen nicht die Löslichkeit von $\mathrm{Li}_{3} \mathrm{CO}_{3}$ wiedergeben, sondern sie sind aus einer Lösung, die $\mathrm{LiOH}$ und $\mathrm{Li}_{2} \mathrm{CO}_{3}$ enthielt, erhalten. Zur Prüfung dieser Annahme wurde ein ganz reines Lithiumcarbonat, das durch Umfällung von $\mathrm{Li}_{2} \mathrm{CO}_{3}$ D.A.B.5 aus salzsaurer Lösung durch Ammoniumearbonat und Trocknen bei $100^{\circ}$ erhalten wurde verwendet. Die Analyse erwies die Reinheit des Salzes.

$$
\mathrm{Li}_{2} \mathrm{CO}_{3} \text {. }
$$

Berechnet: $\mathrm{Li}_{8} \mathrm{O} \quad 40,43 \quad \mathrm{CO}_{2} \quad 59,57 \%$

Gefunden: $\mathrm{Li}_{8} \mathrm{O} \quad 40,33 \quad 40,39 \quad \mathrm{CO}_{2} 59,33 \quad 59,46 \%$

Löslichkeit von $\mathrm{Li}_{2} \mathrm{CO}_{8}$ in Wasger bei $0^{\circ}$.

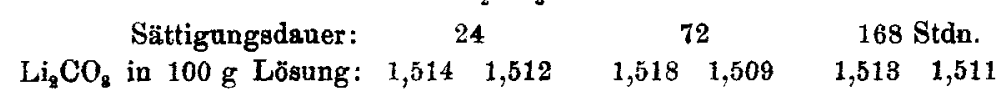

Der Mittelwert 1,513 steht in befriedigender Übereinstimmung mit den Angaben von Bewad.

Um die Einstellung des Gleichgewichtes $\mathrm{LiOH} \rightleftharpoons \mathrm{Li}_{2} \mathrm{CO}_{3}$ in einer siedenden Lithiumcarbonatlösung festzustellen, wurden die folgenden Versuchsreihen angestellt, die Herr Dr. GERHART JANDER im Jahre 1917 ausgeführt hat. Für die Überlassung seiner Zahlenwerte sind wir ihm zu bestem Danke verpflichtet.

Gleichgewichtsermittlung $\mathrm{Li}_{2} \mathrm{CO}_{3} \rightleftharpoons \mathrm{LiOH}$ in siedender Lithiumcarbonatlösung.

\begin{tabular}{|c|c|c|c|c|c|}
\hline $\begin{array}{l}\text { Dauer des } \\
\text { Siedens in } \\
\text { Stunden }\end{array}$ & \begin{tabular}{|} 
Gesamtgehalt \\
der Lösung \\
an Li in \\
$100 \mathrm{~g}$
\end{tabular} & $\begin{array}{l}\text { Gehalt der } \\
\text { Lösung an } \\
\mathrm{LiOH} \text { ing } \mathrm{Li} \\
\text { in } 100 \mathrm{~g}\end{array}$ & $\begin{array}{l}\text { Gehalt der } \\
\text { Lösung an } \\
\mathrm{Li}_{8} \mathrm{CO}_{3} \text { in } \mathrm{g} \\
\mathrm{Li} \text { in } 100 \mathrm{~g}\end{array}$ & $\begin{array}{l}\text { Gehalt der } \\
\text { Lösung an } \\
\mathrm{CO}_{3}^{\prime} \text {-Ionen } \\
\text { in } \mathrm{g} \text { in } 100 \mathrm{~g}\end{array}$ & $\begin{array}{l}\text { Molekular- } \\
\text { quotient: } \\
\mathrm{Li} / \mathrm{CO}_{\mathrm{g}} \text { in der } \\
\text { Lösung }\end{array}$ \\
\hline $\begin{array}{r}1 \\
10 \\
34 \\
82 \\
175 \\
370 \\
\end{array}$ & $\begin{array}{l}0,1178 \\
0,1185 \\
0,1238 \\
0,1228 \\
0,1289 \\
0,1295 \\
\end{array}$ & $\begin{array}{l}0,0025 \\
0,0093 \\
0,0196 \\
0,0307 \\
0,0415 \\
0,0409\end{array}$ & $\begin{array}{l}0,1153 \\
0,1092 \\
0,1042 \\
0,0921 \\
0,0875 \\
0,0886\end{array}$ & $\begin{array}{l}0,4983 \\
0,4720 \\
0,4503 \\
0,3983 \\
0,3778 \\
0,3829\end{array}$ & $\begin{array}{l}2,044 \\
2,171 \\
2,38 \\
2,67 \\
2,95 \\
2,90\end{array}$ \\
\hline $\begin{array}{r}20 \\
48 \\
96 \\
216 \\
408\end{array}$ & $\begin{array}{l}0,1153 \\
0,1179 \\
0,1195 \\
0,1212 \\
0,1281\end{array}$ & $\begin{array}{l}0,0067 \\
0,0152 \\
0,0208 \\
0,0253 \\
0,0408\end{array}$ & $\begin{array}{l}0,1086 \\
0,1027 \\
0,0987 \\
0,0959 \\
0,0872\end{array}$ & $\begin{array}{l}0,4695 \\
0,4438 \\
0,4265 \\
0,4147 \\
0,3795\end{array}$ & $\begin{array}{l}2,123 \\
2,30 \\
2,42 \\
2,53 \\
2,92\end{array}$ \\
\hline
\end{tabular}


In einem Kolben aus Reinnickel wurde die Lithiumcarbonatlösung am Rückflußkühler im Ölbad auf $125-130^{\circ}$ erhitzt. In den entnommenen Proben wurde alkalimetrisch nach bekanntem Verfahren einerseits der Gesamtlithiumgehalt, andererseits der OH-Ionengehalt ermittelt. Die Ergebnisse sind aus der vorstehenden Tabelle ersichtlich, die zwei von fünf ganz gleichartig verlaufenen Versuchsreihen enthält.

Jiese Werte zeigen, daB aus der siedenden $\mathrm{Li}_{2} \mathrm{CO}_{3}$-Lösung $\mathrm{CO}_{2}$ entweicht, bis sich sehr annähernd das Molekularverhältnis $1 \mathrm{Mol}$ $\mathrm{LiOH}$ zu $1 \mathrm{Mol} \mathrm{Li}_{2} \mathrm{CO}_{3}$ eingestellt hat. Bei der Bestimmung der Löslichkeit von $\mathrm{Li}_{2} \mathrm{CO}_{3}$ erhält man mithin, besonders bei höherer Temperatur, keine definierten Werte und die Zahlen von BEwaD können höchstens als die eines Gemenges von $\mathrm{Li}_{2} \mathrm{CO}_{3}$ und $\mathrm{LiOH}$ angesprochen werden. Rechnet man den Gesamtlithiumgehalt der Reihe 5 obiger Tabelle $=0,1295 \mathrm{~g} \mathrm{Li}$ unter der unrichtigen Annahmı, daB das Lithium nur als $\mathrm{Li}_{2} \mathrm{CO}_{3}$ in der Lösung sei, um, so erbält man bei $100^{\circ} 0,685 \mathrm{~g} \mathrm{Li}_{2} \mathrm{CO}_{3}$ in $100 \mathrm{~g}$ Lösung einen Wert, der hinter der Zahl von BEWAD noch erheblich zurückbleibt.

Berlin $\boldsymbol{N}$, Wissenschaftlich-chemisches Laboratorium.

Bei der Redaktion eingegangen am 25. Oktober 1921. 